\title{
A Fourier Approach to Model Electromagnetic Fields Scattered by a Buried Rectangular Cavity
}

\author{
John L. Fleming and Jessica Moser \\ Department of Mathematics and Computer Science, Duquesne University, Pittsburgh, PA 15282, USA \\ Correspondence should be addressed to John L. Fleming, flemingj@duq.edu
}

Received 23 January 2009; Revised 31 August 2009; Accepted 26 October 2009

Recommended by Mohammad Younis

\begin{abstract}
We consider the problem of a two-dimensional rectangular cavity in a PEC half plane covered by layers of material with uniform thickness. The rectangular geometry allows for an application of Fourier methods to solve the problem. The paper will also discuss how to compute the far field scattering once the solution is found. The fast mode matching approximation technique developed by Morgan and Schwering will be applied to this layered cavity problem setting. Numerical results will show that using the Fourier solution on the layered problem combined with Morgan and Schwering's technique can produce good approximations while using much less computation time than would be required for the entire solution.
\end{abstract}

Copyright (C 2009 J. L. Fleming and J. Moser. This is an open access article distributed under the Creative Commons Attribution License, which permits unrestricted use, distribution, and reproduction in any medium, provided the original work is properly cited.

\section{Introduction}

The cavity problem has been extensively studied due to its importance in the computation of radar cross section. A two-dimensional cavity can be used to model long seems or cracks in metallic surfaces which can significantly contribute to the overall radar profile of large objects. The cavity problem has been solved with a variety of methods including integral equations, finite elements, and Fourier methods. These approaches are primarily applied to a problem with a material filled cavity in a PEC ground place opening into an empty half space.

A neglected aspect of the problem is when the cavity is buried beneath a layered material. A two-dimensional cavity beneath a layered material can serve as model for seams and cracks in metallic surfaces which are covered by paint or materials applied during a manufacturing process. The material covering would mean that the cavities are invisible to a visual inspection. However, they may be revealed by understanding the scattering characteristics of the cavity when exposed to an electromagnetic field. The mathematical model can serve as a predictor of the scattering characteristics for use in nondestructive testing. 


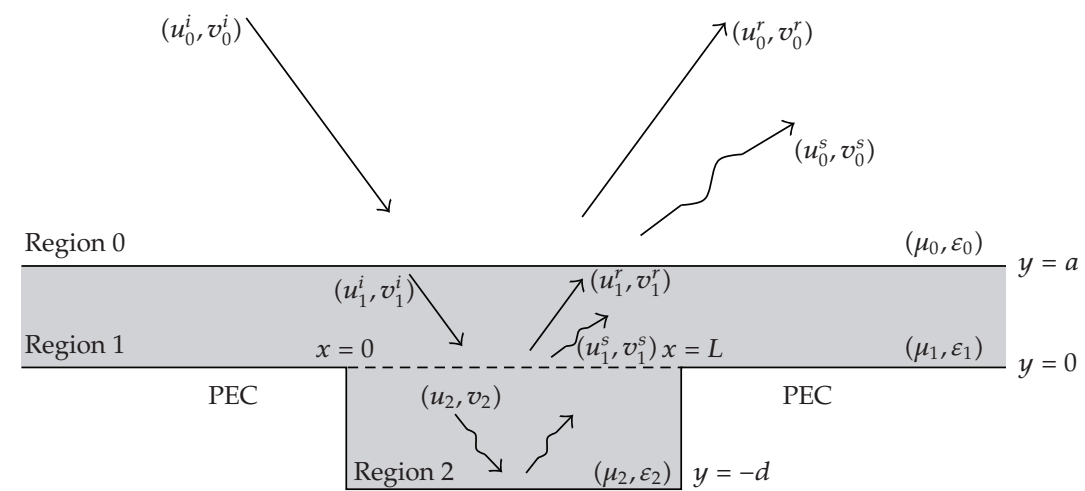

Figure 1: Layered geometry.

We want to compute the fields in the finite cavity region shown in Figure 1. The computation can be done with integral equation methods. The disadvantage of this approach is that a layered Green's function must be used which adds difficulty to the problem and brings up convergence issues [1]. A finite element approach can also be used but would require appropriate boundary conditions at the cavity opening [2]. A key contribution of this manuscript is to provide the understanding of the Fourier approach in the overlayer setting on which an appropriate boundary condition is based.

In addition, the Fourier approach has some unique advantages. It is the sensible and natural approach for a rectangular geometry. Further, it is the only approach which can employ Morgan and Schwering's mode matching method and improved mode matching methods to get very fast approximations for large cavities and high frequencies. Very large problems with high wave numbers will become computationally cumbersome for any technique. Using the fast mode matching approach opens the door to dealing with very large problems which could not be dealt with by other conventional approaches $[3,4]$.

\section{Problem Setting}

We consider a time harmonic plane wave with fixed frequency $\omega$ incident onto a ideal metal or a perfect electrical conductor (PEC) half plane which is covered by a material layer(s) of uniform thickness. Embedded in the PEC half space is a cavity with an opening to the upper half space. As indicated in Figure 1, the region above the material layer is empty space and will be denoted by region 0 , the region inside the material layer will be denoted by region 1 , while the cavity will be called region 2 . The opening of the cavity is the interval $[0, L]$ in the $x$-direction. The depth of the cavity is denoted by $d$ and top opening of the cavity is located at $y=0$ while the bottom of the cavity is at $y=-d$. The cavity may be filled with a uniform material. The electric permittivities and magnetic permeabilities of the materials are denoted by $\epsilon_{k}$ and $\mu_{k}$ where $k=0,1,2$ represents the region. Multiple material layers can be considered for both the inside of the cavity as well as the material covering the PEC half space. The paper will deal exclusively with a single material in both cases. The generalization to multiple layered materials follows easily once a single material is understood. 
We will solve for the electric and magnetic fields denoted by $E$ and $H$ which satisfy Maxwell's equations [5]. In the two-dimensional setting, Maxwell's equations can be separated into two fundamental polarizations [5]. In the transverse magnetic (TM) polarization the electric field has only a $z$ component which will be computed. We denote the $z$ component of $E$ by $u$. The other polarization is the transverse electric (TE) where the magnetic field $H$ has only a $z$ component which is denoted by $v$. In Sections 5 and 6 , we will solve for the unknown functions $u$ and $v$ inside the cavity region. In both cases $u$ and $v$ will satisfy a Helmholtz equation with appropriate boundary conditions. Once the solutions are found in region 2 we will show how to compute far field values.

Note that in regions 0 and 1 the solutions can be decomposed into a superpositions of fields. We use a subscript to denote which region the field is contained. Thus $u_{k}$ and $v_{k}$ for $k=0,1,2$ are the solutions in each of the respective regions. In region 0 , the fields $u_{0}$ and $v_{0}$ are expressed as a sum of an incoming plane waves $u_{0}^{i}$ and $v_{0}^{i}$, outgoing plane waves $u_{0}^{r}$ and $v_{0}^{r}$, and a scattered fields $u_{0}^{s}$ and $v_{0}^{s}$. The incoming waves in region 0 are known and specified. The remaining fields will be computed in following sections below. In region 1 , the fields $u_{1}$ and $v_{1}$ also consist of incoming plane waves $u_{1}^{i}$ and $v_{1}^{i}$, outgoing plane waves $u_{1}^{r}$ and $v_{1}^{r}$ and scattered fields $u_{1}^{s}$ and $v_{0}^{s}$ all of which are unknown. When appropriate we will combine the incoming and outgoing plane waves into a single term $u_{k}^{i r}=u_{k}^{i}+u_{k}^{r}$ and $v_{k}^{i r}=v_{k}^{i}+v_{k}^{r}$ for $k=0,1$. Finally the fields $u_{2}$ and $v_{2}$ in the cavity region 2 will not be decomposed but solved as single fields.

When the known incident plane waves $u_{0}^{i}$ and $v_{0}^{i}$ come in from region 0 they interact with the material layer (region 1). The interaction produces reflected plane waves $u_{0}^{r}$ and $v_{0}^{r}$ as well as transmitted plane waves into region 1 . The transmitted plane waves propagate into region 1 which produces plane waves propagating in the positive $y$-direction $\left(u_{1}^{r}\right.$ and $v_{1}^{r}$ ) and in the negative $y$-direction $\left(u_{1}^{r}\right.$ and $v_{1}^{r}$ ). While the plane waves $u_{0}^{i}$ and $v_{0}^{i}$ are given, the remainder of the plane waves must be computed. They are computed assuming that the cavity (region 2) is not present. Once the plane waves are completely known they are used as a source field which interacts with the cavity to produce scattered fields $u_{k}^{s}$ and $v_{k}^{s}$ with $k=0,1$ in regions 0 and 1 as well as transmitted fields $u_{2}$ and $v_{2}$ in region 2 .

Once the transmitted field in the cavity is computed we want to find far field values based on this solution. The difficulty here is that the cavity does not open to an empty half space. The upper half space is partially filled with material layer. The Fourier representations will allow the solution of the transmitted field in the cavity to be transferred to the upper half space and interpreted appropriately to allow far field computations.

\section{Previous Work}

There have been numerous techniques developed to account for the electromagnetic scattering from a cavity aperture in a ground plane, including Fourier transform, finite element, integral equations, cavity mode coupling and impedance boundary conditions. The solution of the cavity problem when buried underneath uniform layers of material has received much less attention.

The Fourier transform technique was utilized by Park and Eom to examine the TE and TM scattering from an empty rectangular cavity embedded in an infinite ground plane $[6,7]$. The method allowed for a closed form solution to be developed by approximating 
a series solution for the scattered field. It analyzed the effect that filling the rectangular cavity with a dielectric material has on the radar cross section. It did not account for material outside of the cavity. Other techniques have dealt with the same situation by employing Green's functions, integral equations and impedance boundary conditions [8,9]. Since these methods tend to be computationally burdensome as the size of the cavity increases, Morgan and Schwering approached this problem by implementing a mode coupling method, and solved for a rapid approximation technique by extending the cavity walls using propagating waveguide modes [4]. Morgan and Schwering's approach was followed by an improved mode matching technique which employed an asymptotic expansion which converges as the frequency increases [3]. This paper presents a similar methodology to duplicate Morgan and Schwering's fast approximation technique for the cavity underneath the material layer.

The finite element method has the advantage of being able to model arbitrary shaped cavities embedded in a ground plane. Analysis of the TM case of a material filled arbitrarily shaped cavity was analyzed by Wood using a set of scalar integral equations, where Howe's masters thesis expanded this to look at the results of the TE cases [10,11]. An alternate solution to this problem was presented by Van and Wood which coupled the finite element method with Fourier transforms, expressing the results of both the TM and TE case [2]. While these papers provide efficient solutions for an arbitrarily shaped cavity, they are dependent on the ideal situation where the material layer is restricted to the cavity space below the half plane. Wood was able to account for this problem, and extended the research to consider the effect of over filling the cavity space with a dielectric material. This was accomplished by creating an artificial boundary condition on a semicircle inscribing the overfilled material region, and combining a hybrid finite element method with Fourier transforms to solve for the far field scattering [12]. This methodology fails when the entire surface is coated with a material since no semicircle can contain the entire material layer.

The work proposed in this paper is an extension of previously published literature, where we are considering an embedded rectangular cavity, in which the entire surface is coated with a dielectric material. Understanding the problem with the restricted cavity shape allows for the boundary conditions to be appropriately defined so that future research can investigate the use of the finite element method to solve for the scattering based on any arbitrary cavity.

\section{Focus of the Paper}

In this paper we focus on modifying the standard rectangular cavity model to accommodate uniform material layers over the cavity opening. The modification of the Fourier approach has intrinsic value as a computational model of a buried rectangular cavity but it also is important since it can be applied to finite element formulation for electromagnetic scattering by nonrectangular buried cavities in future work.

In addition to providing the layered modification to the standard Fourier approach the paper will also demonstrate that the fast approximation method used by Morgan and Schwering and extended by Bao and Zhang, works well with the modification $[3,4]$. The fast approximations are very important for electrically large cavities which take a lot of computation time. Also, the fast approximation can be used for inverse and design problems where solutions must be computed many times over as parameters are determined in order optimize scattering characteristics. 


\section{Overview}

The two-dimensional cavity problem can be split into two fundamental polarizations. The transverse magnetic (TM) polarization where $E=(0,0, u)$ and the transverse electric (TE) polarization where $H=(0,0, v)$. In both cases we find the $z$-component of the field in question as a solution. In general the fields satisfy Maxwell's equations, but these can easily be reduced to the scalar Helmholtz equation for both polarizations. The difference between the two polarizations is the boundary conditions which will be clarified in the following sections.

Much of the calculations to follow rely on enforcing the continuity or boundary conditions. The continuity conditions for electromagnetic fields state that the tangential components of the electric and magnetic field are continuous at interfaces. Thus we have that $n \times E_{0}=n \times E_{1}$ and $n \times H_{0}=n \times H_{1}$ where $n$ is the normal vector. At the interfaces between regions 0 and 1 and between regions 1 and 2 we will have $n=(0,1,0)$. Note that $E_{0}$ and $E_{1}$ are the electric fields in regions 0 and 1 while $H_{0}$ and $H_{1}$ are the magnetic fields in regions 0 and 1 . The same continuity holds for the interface between regions 1 and 2 for the fields $E_{1}, E_{2}, H_{1}$ and $H_{2}$.

Note that inside a PEC material we have $E=0$. Therefore when enforcing the continuity conditions we get that $E \times n=0$ at the boundary of any PEC material. For the TM polarization this means $u=0$ at the boundary of the PEC material. For the TE case we have by applying Maxwell's equations that $(1 / \epsilon)(d v / d n)=0$. In both cases we will solve for $u_{2}$ and $v_{2}$ in region 2 . The appropriate boundary conditions at the walls of the cavity will be enforced along with the continuity conditions at the interfaces between regions 0 and 1 and regions 1 and 2.

For the TM case the continuity conditions of the electric field simply states that $u_{0}=u_{1}$ at $y=a$ and $u_{1}=u_{2}$ at $y=0$. The magnetic continuity condition reduces to $\left(1 / \mu_{0}\right)\left(\partial u_{0} / \partial y\right)=$ $\left(1 / \mu_{1}\right)\left(\partial u_{1} / \partial y\right)$ at $y=a$ and $\left(1 / \mu_{1}\right)\left(\partial u_{1} / \partial y\right)=\left(1 / \mu_{2}\right)\left(\partial u_{2} / \partial y\right)$ at $y=0$. For the TE case the continuity conditions of the magnetic field simply states that $v_{0}=v_{1}$ at $y=a$ and $v_{1}=v_{2}$ at $y=0$. The electric continuity condition reduces to $\left(1 / \epsilon_{0}\right)\left(\partial v_{0} / \partial y\right)=\left(1 / \epsilon_{1}\right)\left(\partial v_{1} / \partial y\right)$ at $y=a$ and $\left(1 / \epsilon_{1}\right)\left(\partial v_{1} / \partial y\right)=\left(1 / \epsilon_{2}\right)\left(\partial v_{2} / \partial y\right)$ at $y=0$.

\section{TM Case}

The TM polarization has only a $z$-component for the electric field $E=(0,0, u)$. In region 0 $u$ is denoted by $u_{0}$ consists of the incident/reflected plane waves $u_{0}^{i}$ and $u_{0}^{r}$ as well as the scattered field $u_{0}^{s}$. Thus we have $u_{0}=u_{0}^{i}+u_{0}^{r}+u_{0}^{s}$. In region $1, u$ is denoted by $u_{1}$ and is again a combination of an incoming and outgoing plane wave along with a scattered field. Therefore $u_{1}=u_{1}^{i}+u_{1}^{r}+u_{1}^{s}$. The transmitted field in region 2 , is denoted by $u_{2}$ but it will not be decomposed into a superposition of fields. It will be found in the cavity by appropriately specifying the incoming and outgoing plane waves in the upper half space with the material over layer (regions 0 and 1) and by using Fourier theory to represent the scattered and transmitted fields in all three regions.

By enforcing continuity conditions at the interfaces along with the boundary conditions at the cavity walls, the transmitted field $u_{2}$ will be determined. At the PEC walls we will have $u_{2}=0$. At the opening to the upper half space we must enforce continuity with a representation of the scattered field $u^{s}$ combined with the incident and reflected fields which captures the effect of the material layer in region 0 . Once all of the boundary conditions are enforced we will be able to determine the solution $u_{2}$. 
The solution $u_{2}$ satisfies the Helmholtz equation $\Delta u_{2}+k^{2} u_{2}=0$. The PEC boundary condition $u_{2}=0$ is enforced at $x=0, x=L$ and $y=-d$. At $y=0$ the continuity conditions are enforced which give the final boundary condition. Note that $k$ is known as the "wave number" and is given by $k=\omega^{2} \epsilon \mu$.

The Helmholtz equation and all of the boundary conditions dictate the type of Fourier representation we use for the various fields. Once we have the appropriate representation and enforce the continuity conditions we can solve for the desired solution $u_{2}$.

\subsection{TM: Incoming and Outgoing Plane Waves}

When dealing with a cavity problem without a material covering an incident plane wave is introduced along with a reflected plane wave both of which are as if no cavity is present. The reflected field is easily computed by enforcing the PEC boundary condition at $y=0$. The scattered field is then viewed as a perturbation of these fields when the cavity is included in the geometry. In this paper, when dealing with a material coating, the incoming and reflected waves increase in complexity because of the layer in region 1 . There will be plane waves propagating in both the positive and negative $y$ directions inside region 0 and region 1 . We denote the superposition of these forward and backward propagating plane waves by $u_{0}^{i r}$ and $u_{1}^{i r}$ in regions 0 and 1 respectively. The incident plane wave $u_{0}^{i}$ in region 0 is assumed to be given. The remaining plane waves are calculated by enforcing the continuity conditions at $y=0$ and $y=a$. In region 0 we have $u_{0}^{i r}=u_{0}^{i}(x, y)+u_{0}^{r}(x, y)$ and in region 1 we have $u_{1}^{i r}=u_{1}^{i}(x, y)+u_{1}^{r}(x, y)$ :

$$
\begin{aligned}
& u_{0}^{i}(x, y)=e^{\imath k_{x}^{0} x-\imath k_{y}^{0} y}, \\
& u_{0}^{r}(x, y)=A_{\mathrm{TM}}^{-} e^{\imath k_{x}^{0} x+\imath k_{y}^{0} y}, \\
& u_{1}^{i}(x, y)=B_{\mathrm{TM}}^{+} e^{\imath k_{x}^{1} x-\imath k_{y}^{1} y}, \\
& u_{1}^{r}(x, y)=B_{\mathrm{TM}}^{-} e^{\imath k_{x}^{1} x+\imath k_{y}^{1} y},
\end{aligned}
$$

where $k_{x}^{0}$ and $k_{y}^{0}$ are related to the wavenumber of region 0 by $\left(k_{x}^{0}\right)^{2}+\left(k_{y}^{0}\right)^{2}=k_{0}^{2}$. The same relationship holds for $k_{x}^{1}$ and $k_{y}^{1}$ in region 1 where we have $\left(k_{x}^{1}\right)^{2}+\left(k_{y}^{1}\right)^{2}=k_{1}^{2}$.

The PEC boundary condition states that $u_{1}^{i r}=0$ at $y=0$. Enforcing this boundary condition allows us to eliminate one of the unknown coefficients

$$
\begin{gathered}
u_{1}^{\imath r}(x, 0)=0, \\
u_{1}^{i}(x, 0)+u_{1}^{r}(x, 0)=0, \\
B_{\mathrm{TM}}^{+} e^{\imath k_{x}^{1} x}=-B_{\mathrm{TM}}^{-} e^{\imath k_{x}^{1} x}, \\
B_{\mathrm{TM}}^{+}=-B_{\mathrm{TM}}^{-} .
\end{gathered}
$$

In order to solve for the remaining unknown coefficients representing the incoming and outgoing plane waves in regions 0 and 1 , the boundary condition of the material interface 
at $y=a$ must be enforced. Applying the electric field continuity condition yields

$$
\begin{aligned}
u_{0}^{i r}(x, a) & =u_{1}^{i r}(x, a), \\
u_{0}^{i}(x, a)+u_{0}^{r}(x, a) & =u_{1}^{i}(x, a)+u_{1}^{r}(x, a), \\
e^{\imath k_{x}^{0} x-i k_{y}^{0} a}+A_{\mathrm{TM}}^{-} e^{\imath k_{x}^{0} x+\imath k_{y}^{0} a}= & B_{\mathrm{TM}}^{+}\left(e^{\imath k_{x}^{1} x-\imath k_{y}^{1} a}-e^{\imath k_{x}^{1} x+\imath k_{y}^{1} a}\right) .
\end{aligned}
$$

Enforcing the magnetic field continuity condition gives

$$
\begin{aligned}
& \frac{1}{\mu_{0}} \frac{\partial u_{0}^{i r}}{\partial y}(x, a)=\frac{1}{\mu_{1}} \frac{\partial u_{1}^{i r}}{\partial y}(x, a), \\
& \mu_{1} \frac{\partial u_{0}^{i}}{\partial y}(x, a)+\mu_{1} \frac{\partial u_{0}^{r}}{\partial y}(x, a)=\mu_{0} \frac{\partial u_{1}^{i}}{\partial y}(x, a)+\mu_{0} \frac{\partial u_{1}^{r}}{\partial y}(x, a), \\
&-\imath k_{y}^{0} \mu_{1} e^{\imath k_{x}^{0} x-\imath k_{y}^{0} a}+\imath k_{y}^{0} \mu_{1} A_{\mathrm{TM}}^{-} e^{\imath k_{x}^{0} x+\imath k_{y}^{0} a}=-\imath k_{y}^{1} \mu_{0} B_{\mathrm{TM}}^{+}\left(e^{i k_{x}^{1} x-\imath k_{y}^{1} a}+e^{\imath k_{x}^{1} x+\imath k_{y}^{1} a}\right) .
\end{aligned}
$$

Combining the above equations gives a system of which can easily be solved by using Cramer's rule:

$$
\begin{gathered}
A_{\mathrm{TM}}^{-}=\frac{e^{-2 \imath k_{y}^{0} a}\left(k_{y}^{0} \mu_{r 1}\left(e^{-\imath k_{y}^{1} a}-e^{\imath k_{y}^{1} a}\right)-k_{y}^{1}\left(e^{-\imath k_{y}^{1} a}+e^{\imath k_{y}^{1} a}\right)\right)}{k_{y}^{1}\left(e^{-\imath k_{y}^{1} a}+e^{\imath k_{y}^{1} a}\right)+k_{y}^{0} \mu_{r 1}\left(e^{-\imath k_{y}^{1} a}-e^{\imath k_{y}^{1} a}\right)}, \\
B_{\mathrm{TM}}^{+}=\frac{2 k_{y}^{0} \mu_{r 1} e^{-\imath k_{y}^{0} a}}{k_{y}^{1}\left(e^{-\imath k_{y}^{1} a}+e^{\imath k_{y}^{1} a}\right)+k_{y}^{0} \mu_{r 1}\left(e^{-\imath k_{y}^{1} a}-e^{\imath k_{y}^{1} a}\right)} .
\end{gathered}
$$

Note that for multiple layers the same approach works in principle but the system of equations will be larger. Each layer will have a set of unknown coefficients and the continuity conditions would be enforced at each interface.

\subsection{TM: Scattered and Transmitted Fields}

We now consider the incoming and outgoing plane waves described above as a source field impinging on the rectangular cavity in region 2 embedded in an infinite PEC ground plane that has been entirely coated with a dielectric material in region 1 . These plane waves already satisfy the Helmholtz equation. We can add in the scattered fields to these plane waves by the superposition principle. The scattered fields are produced by the interaction of the plane waves with the cavity opening. The interaction produces scattered fields in regions 1 and 0 and a transmitted field in region 2. The scattered and transmitted fields will satisfy the Helmholtz equation as well as the same electric and magnetic continuity conditions. 
Since the scattered field in region 0 is not bounded above in the $y$-direction, it consists of only an outgoing field. Therefore, the Fourier representation is

$$
u_{0}^{s}(x, y)=\int_{-\infty}^{\infty} D_{\mathrm{TM}}(\lambda) e^{-\alpha_{0} y} e^{2 \pi \imath \lambda x} d \lambda
$$

where $\alpha_{0}=\sqrt{(2 \pi \lambda)^{2}-k_{0}^{2}}$.

Since region 1 is bounded both above and below, the scattered field can propagate in both the positive and negative $y$-directions. The representation of the scattered field in region 1 is given by

$$
u_{1}^{s}(x, y)=\int_{-\infty}^{\infty}\left(G_{\mathrm{TM}}(\lambda) e^{-\alpha_{1} y}+H_{\mathrm{TM}}(\lambda) e^{\alpha_{1} y}\right) e^{2 \pi \imath \lambda x} d \lambda,
$$

where $\alpha_{1}=\sqrt{(2 \pi \lambda)^{2}-k_{1}^{2}}$. Note that both representations are solutions to the Helmholtz equation but that the unknown Fourier transforms $D_{\mathrm{TM}}(\lambda), G_{\mathrm{TM}}(\lambda)$ and $H_{\mathrm{TM}}(\lambda)$ have not been specified.

The transmitted field in region 2 has compact support and must satisfy the PEC boundary conditions at the cavity walls. Thus, the Fourier representation of the transmitted field is given by

$$
u_{2}(x, y)=\sum_{n=1}^{\infty} A_{n}^{\mathrm{TM}} \sin \left(\frac{n \pi}{L} x\right) \sinh \left(\gamma_{2}(y+d)\right)
$$

where $\gamma_{2}=\sqrt{(n \pi / L)^{2}-k_{2}^{2}}$. The unknown Fourier coefficients $A_{n}$ will be found approximately. In order to solve for the coefficients the unknown functions $D_{\mathrm{TM}}(\lambda), G_{\mathrm{TM}}(\lambda)$ and $H_{\mathrm{TM}}(\lambda)$ must be eliminated by using the continuity conditions at $y=0$ and $y=a$. We must enforce the continuity conditions at $y=0$ and at $y=a$. Once the conditions are enforced we can reduce the problem to a system of equations for the unknown Fourier coefficients $A_{n}$ 's.

\subsection{Boundary $y=a$}

By first applying the electric field continuity we are able to solve for the transform $D_{\mathrm{TM}}(\lambda)$ :

$$
\begin{aligned}
u_{0}(x, a) & =u_{1}(x, a) \\
u_{0}^{i r}(x, a)+u_{0}^{s}(x, a) & =u_{1}^{i r}(x, a)+u_{1}^{s}(x, a) .
\end{aligned}
$$

Since $u^{i r}$ already satisfies the continuity conditions we have $u_{0}^{s}(x, a)=u_{1}^{s}(x, a)$. 

have

Now going to the Fourier representations of the scattered field in regions 0 and 1 we

$$
\begin{aligned}
\int_{-\infty}^{\infty} D_{\mathrm{TM}}(\lambda) e^{-\alpha_{0} a} e^{2 \pi \lambda x} d \lambda & =\int_{-\infty}^{\infty}\left(G_{\mathrm{TM}}(\lambda) e^{-\alpha_{1} a}+H_{\mathrm{TM}}(\lambda) e^{\alpha_{1} a}\right) e^{2 \pi \imath \lambda x} d \lambda, \\
\mathcal{F}^{-1}\left(D_{\mathrm{TM}}(\lambda) e^{-\alpha_{0} a}\right) & =\mathcal{F}^{-1}\left(G_{\mathrm{TM}}(\lambda) e^{-\alpha_{1} a}+H_{\mathrm{TM}}(\lambda) e^{\alpha_{1} a}\right), \\
D_{\mathrm{TM}}(\lambda) e^{-\alpha_{0} a} & =G_{\mathrm{TM}}(\lambda) e^{-\alpha_{1} a}+H_{\mathrm{TM}}(\lambda) e^{\alpha_{1} a} .
\end{aligned}
$$

Next, applying the boundary constraints controlling for the continuity of the magnetic field at $y=a$ also yields a solution for the coefficient $D_{\mathrm{TM}}(\lambda)$ :

$$
\mu_{1} \frac{\partial u_{0}^{i r}}{\partial y}(x, a)+\mu_{1} \frac{\partial u_{0}^{s}}{\partial y}(x, a)=\mu_{0} \frac{\partial u_{1}^{i r}}{\partial y}(x, a)+\mu_{0} \frac{\partial u_{1}^{s}}{\partial y}(x, a)
$$

Again, knowing that $u^{i r}$ already satisfies the magnetic continuity conditions we have

$$
\mu_{1} \frac{\partial u_{0}^{s}}{\partial y}(x, a)=\mu_{0} \frac{\partial u_{1}^{s}}{\partial y}(x, a)
$$

Returning to the Fourier representations we have that

$$
\begin{aligned}
-\mu_{1} \int_{-\infty}^{\infty} D_{\mathrm{TM}}(\lambda) \alpha_{0} e^{-\alpha_{0} a} e^{2 \pi \imath \lambda x} d \lambda & =\mu_{0} \int_{-\infty}^{\infty} \alpha_{1}\left(H_{\mathrm{TM}}(\lambda) e^{\alpha_{1} a}-G_{\mathrm{TM}}(\lambda) e^{-\alpha_{1} a}\right) e^{2 \pi \imath \lambda x} d \lambda \\
-\mu_{1} \mathcal{F}^{-1}\left(\alpha_{0} D_{\mathrm{TM}}(\lambda) e^{-\alpha_{0} a}\right) & =\mu_{0} \mathcal{F}^{-1}\left(\alpha_{1}\left(H_{\mathrm{TM}}(\lambda) e^{\alpha_{1} a}-G_{\mathrm{TM}}(\lambda) e^{-\alpha_{1} a}\right)\right) \\
-\mu_{1} \alpha_{0} D_{\mathrm{TM}}(\lambda) e^{-\alpha_{0} a} & =\mu_{0} \alpha_{1} H_{\mathrm{TM}}(\lambda) e^{\alpha_{1} a}-\mu_{0} \alpha_{1} G_{\mathrm{TM}}(\lambda) e^{-\alpha_{1} a}
\end{aligned}
$$

The results from the boundary condition at $y=a$ then provide a system of equations that can be solved using Cramer's rule. In general, if there were multiple layers the system of equations would be larger and the continuity conditions would be enforced at each material interface. We solve the system to get the unknown functions $G_{\mathrm{TM}}(\lambda)$ and $H_{\mathrm{TM}}(\lambda)$ written in terms of $D_{\mathrm{TM}}(\lambda)$ :

$$
\begin{aligned}
& G_{\mathrm{TM}}(\lambda)=D_{\mathrm{TM}}(\lambda) e^{-\alpha_{0} a}\left(\frac{e^{\alpha_{1} a}\left(\mu_{0} \alpha_{1}+\mu_{1} \alpha_{0}\right)}{2 \mu_{0} \alpha_{1}}\right) \\
& H_{\mathrm{TM}}(\lambda)=D_{\mathrm{TM}}(\lambda) e^{-\alpha_{0} a}\left(\frac{e^{-\alpha_{1} a}\left(\mu_{0} \alpha_{1}-\mu_{1} \alpha_{0}\right)}{2 \mu_{0} \alpha_{1}}\right) .
\end{aligned}
$$

We now want to eliminate $D_{\mathrm{TM}}(\lambda)$ from the problem to be left with only the Fourier coefficients $A_{n}$ 's of the transmitted field $u_{2}$ as unknowns. Further, these coefficients will be the solution to a system of equations which we will solve to get our approximate solution. 


\subsection{Boundary $y=0$}

We use the continuity conditions once again for $u_{1}$ and $u_{2}$ at the interface between regions 1 and 2. First the electric field continuity gives

$$
\begin{gathered}
u_{1}(x, 0)=u_{2}(x, 0), \\
u_{1}^{i r}(x, 0)+u_{1}^{s}(x, 0)=u_{2}(x, 0) .
\end{gathered}
$$

Since $u^{i r}=0$ at $y=0$ we have

$$
u_{1}^{s}(x, 0)=u_{2}(x, 0)
$$

Using the Fourier representations we have

$$
\begin{gathered}
\int_{-\infty}^{\infty}\left(G_{\mathrm{TM}}(\lambda)+H_{\mathrm{TM}}(\lambda)\right) e^{2 \pi \imath \lambda x} d \lambda=\sum_{n=1}^{\infty} A_{n}^{\mathrm{TM}} \sin \left(\frac{n \pi}{L} x\right) \sinh \left(\gamma_{2} d\right), \\
\mathcal{F}^{-1}\left(G_{\mathrm{TM}}(\lambda)+H_{\mathrm{TM}}(\lambda)\right)=\sum_{n=1}^{\infty} A_{n}^{\mathrm{TM}} \sin \left(\frac{n \pi}{L} x\right), \\
G_{\mathrm{TM}}(\lambda)+H_{\mathrm{TM}}(\lambda)=\sum_{n=1}^{\infty} A_{n}^{\mathrm{TM}} \mp\left(\sin \left(\frac{n \pi}{L} x\right)\right) .
\end{gathered}
$$

Substitute in the solution for $G_{\mathrm{TM}}(\lambda)$ and $H_{\mathrm{TM}}(\lambda)$, into the equation to get

$$
D_{\mathrm{TM}}(\lambda) e^{-\alpha_{0} a}\left(\frac{\mathcal{Q}_{A}^{\mathrm{TM}}}{2 \mu_{0} \alpha_{1}}\right)=\sum_{n=1}^{\infty} A_{n}^{\mathrm{TM}} \mathcal{F}\left(\sin \left(\frac{n \pi}{L} x\right)\right)
$$

and solving for $D_{\mathrm{TM}}(\lambda)$ yields:

$$
D_{\mathrm{TM}}(\lambda) e^{-\alpha_{0} a}=\frac{2 \mu_{0} \alpha_{1} \sum_{n=1}^{\infty} A_{n}^{\mathrm{TM}} \mp(\sin ((n \pi / L) x))}{\mathcal{Q}_{A}^{\mathrm{TM}}},
$$

where

$$
\mathcal{Q}_{A}^{\mathrm{TM}}=e^{-\alpha_{1} a}\left(\mu_{0} \alpha_{1}-\mu_{1} \alpha_{0}\right)+e^{\alpha_{1} a}\left(\mu_{0} \alpha_{1}+\mu_{1} \alpha_{0}\right) .
$$

Next, the continuity of the magnetic field across the cavity opening at $y=0$ gives

$$
\begin{gathered}
\frac{-1}{\imath w \mu_{1}} \frac{\partial u_{1}}{\partial y}(x, 0)=\frac{-1}{\imath w \mu_{2}} \frac{\partial u_{2}}{\partial y}(x, 0) \\
\mu_{2} \frac{\partial u_{1}^{i r}}{\partial y}(x, 0)+\mu_{2} \frac{\partial u_{1}^{s}}{\partial y}(x, 0)=\mu_{1} \frac{\partial u_{2}}{\partial y}(x, 0) .
\end{gathered}
$$


Using the Fourier representations we have

$$
\begin{gathered}
-2 \imath k k_{y}^{1} \mu_{2} B_{\mathrm{TM}}^{+} e^{\imath k_{x}^{1} x}+\mu_{2} \int_{-\infty}^{\infty} \alpha_{1}\left(H_{\mathrm{TM}}(\lambda)-G_{\mathrm{TM}}(\lambda)\right) e^{2 \pi \imath \imath x} d \lambda \\
=\mu_{1} \sum_{n=1}^{\infty} A_{n}^{\mathrm{TM}} \gamma_{2} \sin \left(\frac{n \pi}{L} x\right) \cosh \left(\gamma_{2} d\right), \\
-2 \imath k_{y}^{1} \mu_{2} B_{\mathrm{TM}}^{+} e^{\imath k_{x}^{1} x}+\mu_{2} \mathcal{F}^{-1}\left(\alpha_{1}\left(H_{\mathrm{TM}}(\lambda)-G_{\mathrm{TM}}(\lambda)\right)\right) \\
=\mu_{1} \sum_{n=1}^{\infty} A_{n}^{\mathrm{TM}} \gamma_{2} \sin \left(\frac{n \pi}{L} x\right) \operatorname{coth}\left(\gamma_{2} d\right) .
\end{gathered}
$$

Again, substitute in for $G_{\mathrm{TM}}(\lambda)$ and $H_{\mathrm{TM}}(\lambda)$ resulting in

$$
\begin{gathered}
-2 \imath k_{y}^{1} \mu_{2} B_{\mathrm{TM}}^{+} e^{\imath k_{x}^{1} x}+\mu_{2} \mathcal{F}^{-1}\left(\alpha_{1}\left(D_{\mathrm{TM}}(\lambda) e^{-\alpha_{0} a} \frac{Q_{B}^{\mathrm{TM}}}{2 \mu_{0} \alpha_{1}}\right)\right) \\
=\mu_{1} \sum_{n=1}^{\infty} A_{n}^{\mathrm{TM}} \gamma_{2} \sin \left(\frac{n \pi}{L} x\right) \operatorname{coth}\left(\gamma_{2} d\right),
\end{gathered}
$$

where

$$
Q_{B}^{\mathrm{TM}}=e^{-\alpha_{1} a}\left(\mu_{0} \alpha_{1}-\mu_{1} \alpha_{0}\right)-e^{\alpha_{1} a}\left(\mu_{0} \alpha_{1}+\mu_{1} \alpha_{0}\right) .
$$

Using the orthogonality of the sine terms this equation can be reduced to.

$$
\begin{aligned}
& -2 \imath k_{y}^{1} \mu_{2} B_{\mathrm{TM}}^{+} \int_{0}^{L} e^{\imath k_{x}^{1} x} \sin \left(\frac{m \pi}{L} x\right) d x \\
& \quad+\mu_{2} \int_{0}^{L} \mathcal{F}^{-1}\left(D_{\mathrm{TM}}(\lambda) e^{-\alpha_{0} a} \frac{Q_{B}^{\mathrm{TM}}}{2 \mu_{0}}\right) \sin \left(\frac{m \pi}{L} x\right) d x=\mu_{1} \frac{L}{2} A_{m}^{\mathrm{TM}} \gamma_{2 m} \operatorname{coth}\left(\gamma_{2 m} d\right) .
\end{aligned}
$$

Then by performing integration and utilizing Parseval's Theorem, our results show.

$$
\begin{aligned}
& -k_{y}^{1} \mu_{2} B_{\mathrm{TM}}^{+}\left(\frac{e^{\imath k_{x}^{1}+\imath m \pi}-1}{\imath k_{x}^{1}+(\imath m \pi / L)}+\frac{1-e^{\imath k_{x}^{1}-\imath m \pi}}{\imath k_{x}^{1}-(\imath m \pi / L)}\right) \\
& \quad+\mu_{2} \int_{-\infty}^{\infty}\left(D_{\mathrm{TM}}(\lambda) e^{-\alpha_{0} a} \frac{\mathcal{Q}_{B}^{\mathrm{TM}}}{2 \mu_{0}}\right) \overline{\mathcal{F}\left(\sin \left(\frac{m \pi}{L} x\right)\right)} d \lambda=\mu_{1} \frac{L}{2} A_{m}^{\mathrm{TM}} \gamma_{2 m} \operatorname{coth}\left(\gamma_{2 m} d\right) .
\end{aligned}
$$


Lastly, the values for $D_{\mathrm{TM}}(\lambda)$ can be substituted in, and rearranging the equation results in:

$$
\begin{aligned}
\sum_{n=1}^{\infty} A_{n}^{\mathrm{TM}} K_{m, n}-\frac{\mu_{1}}{\mu_{2}} \frac{L}{2} A_{m}^{\mathrm{TM}} \gamma_{2 m} \operatorname{coth}\left(\gamma_{2 m} d\right) \\
=k_{y}^{1} B_{\mathrm{TM}}^{+}\left(\frac{e^{\imath k_{x}^{1}+\imath m \pi}-1}{\imath k_{x}^{1}+\imath m \pi / L}+\frac{1-e^{\imath k_{x}^{1}-\imath m \pi}}{\imath k_{x}^{1}-\imath m \pi / L}\right),
\end{aligned}
$$

where

$$
K_{m, n}=\int_{-\infty}^{\infty} \alpha_{1} \frac{Q_{B}^{\mathrm{TM}}}{\mathcal{Q}_{A}^{\mathrm{TM}}} \mathcal{F}\left(\sin \left(\frac{n \pi}{L} x\right)\right) \overline{\mathcal{F}\left(\sin \left(\frac{m \pi}{L} x\right)\right)} d \lambda
$$

The Fourier coefficients, $A_{n}^{\mathrm{TM}}$, satisfy an infinite system of linear equations. The solution can be approximated computationally by truncating the infinite sum to a finite number of terms. Typically, the number of terms should be at least large enough to include all of the "guided modes." The convergence issues around truncating such an infinite systems of equations are not well understood but a partial discussion is given in [13]. This solution of the finite system is used as an approximate set of Fourier coefficients for the representation of $u_{2}$ in the cavity.

\section{TE Case}

We now look at the transverse electric case where assume $H=(0,0, v)$. The approach is much the same as the TM case. The unknown function will satisfy the Helmholtz equation $\Delta v+k^{2} v=0$. The primary difference is the enforcement of the PEC boundary condition. Since we are now solving for the $z$ component of the magnetic field we must account for how the PEC boundary condition is represented. If we have $H=(0,0, v)$ we can apply Maxwell's equations to produce $E$. We can then compute the tangential component of $E$ and set it equal to zero. Doing so for the TE case gives us that $(1 / \epsilon)(d v / d n)=0$. That is the normal derivative of $v$ will be equal to zero. This condition must be enforced in region 1 when we compute the incident and reflected field and also in region 2 when we represent the transmitted field in the cavity.

\subsection{TE: Incoming and Outgoing Plane Waves}

For the TE case we are assuming that we have a TE-polarized incident magnetic field. The reflections and transmissions at the material interface and at the PEC boundary will produce forward and backward propagating plane waves in both regions. We can solve for the these fields $v_{0}^{i r}$ and $v_{1}^{i r}$ in regions 0 and 1 just as we did in the TM case. The only difference in 
the PEC boundary condition at $y=0$ now is applied to the normal derivative of $v$ instead of $v$ itself. We have the following:

$$
\begin{gathered}
v^{i, 0}(x, y)=e^{\imath k_{x}^{0} x-\imath k_{y}^{0} y}, \\
v^{r, 0}(x, y)=A_{\mathrm{TE}}^{-} e^{\imath k_{x}^{0} x+\imath k_{y}^{0} y}, \\
v^{i, 1}(x, y)=B_{\mathrm{TE}}^{+} e^{\imath k_{x}^{1} x-\imath k_{y}^{1} y}, \\
v^{r, 1}(x, y)=B_{\mathrm{TE}}^{-} e^{\imath k_{x}^{1} x+\imath k_{y}^{1} y} .
\end{gathered}
$$

These results provide us a system of equations in two unknowns that can be solved using Cramer's rule to give

$$
\begin{gathered}
A_{\mathrm{TE}}^{-}=\frac{e^{-2 \imath k_{y}^{0} a}\left(-k_{y}^{1}\left(e^{\imath k_{y}^{1} a}-e^{-\imath k_{y}^{1} a}\right)-k_{y}^{0} \varepsilon_{r 1}\left(e^{-\imath k_{y}^{1} a}+e^{\imath k_{y}^{1} a}\right)\right)}{k_{y}^{1}\left(e^{\imath k_{y}^{1} a}-e^{-\imath k_{y}^{1} a}\right)-k_{y}^{0} \varepsilon_{r 1}\left(e^{-\imath k_{y}^{1} a}+e^{\imath k_{y}^{1} a}\right)}, \\
B_{\mathrm{TE}}^{+}=\frac{-2 k_{y}^{0} \varepsilon_{r 1} e^{-\imath k_{y}^{0} a}}{k_{y}^{1}\left(e^{\imath k_{y}^{1} a}-e^{-\imath k_{y}^{1} a}\right)-k_{y}^{0} \varepsilon_{r 1}\left(e^{-\imath k_{y}^{1} a}+e^{\imath k_{y}^{1} a}\right)} .
\end{gathered}
$$

As in the TM case, these incoming and outgoing plane waves in regions 0 and 1 are used as a source field. The plane waves impinge on the cavity in region 2 and produce scattered fields in regions 0 and 1 along with a transmitted field inside region 2 .

\subsection{TE: Scattered and Transmitted Fields}

We now consider the TE case when an incident plane wave impinges on a rectangular cavity embedded in an infinite ground plane that has been entirely coated with a dielectric material. The incoming and outgoing plane waves of regions 0 and 1 were found above. Now the embedded cavity requires representations for the transmitted and scattered fields. The representation of the scattered field in region 0 is

$$
v_{0}^{s}(x, y)=\int_{-\infty}^{\infty} D_{\mathrm{TE}}(\lambda) e^{-\alpha_{0} y} e^{2 \pi \imath \lambda x} d \lambda
$$

Since region 1 is bounded both above and below, and the scattered filed propagates in both the positive and negative $y$ direction, we must account for this using the following Fourier representation:

$$
v_{1}^{s}(x, y)=\int_{-\infty}^{\infty}\left(G_{\mathrm{TE}}(\lambda) e^{-\alpha_{1} y}+H_{\mathrm{TE}}(\lambda) e^{\alpha_{1} y}\right) e^{2 \pi \imath \lambda x} d \lambda
$$


Lastly, the representation of the transmitted field is

$$
v_{2}(x, y)=\sum_{n=0}^{\infty} A_{n}^{\mathrm{TE}} \cos \left(\frac{n \pi}{L} x\right) \cosh \left(\gamma_{2}(y+d)\right)
$$

Note that this Fourier representation is chosen to satisfy the PEC boundary condition when it is enforced on the normal derivative.

The continuity conditions will again be used at $y=a$ to solve for the unknown functions $G_{\mathrm{TE}}(\lambda)$ and $H_{\mathrm{TE}}(\lambda)$ in terms of $D_{\mathrm{TE}}(\lambda)$. Once this is done the continuity conditions are enforced at $y=0$ to eliminate $D_{\mathrm{TE}}(\lambda)$ from the problem and to be left with only the unknown Fourier coefficients for $v_{2}$. Using orthogonality and Parseval's theorem these coefficients are shown to satisfy an infinite system of equations:

$$
\begin{aligned}
& B_{\mathrm{TE}}^{+}\left(\frac{e^{\imath k_{x}^{1} L+\imath m \pi}-1}{\imath k_{x}^{1}+\imath m \pi / L}+\frac{e^{\imath k_{x}^{1} L-\imath m \pi}-1}{\imath k_{x}^{1}-\imath m \pi / L}\right) \\
&=-\frac{\varepsilon_{1}}{\varepsilon_{2}} \sum_{n=0}^{\infty} A_{n}^{\mathrm{TE}} \gamma_{2} L_{m, n}+ \begin{cases}A_{m}^{\mathrm{TE}} L \operatorname{coth}\left(\gamma_{2 m} d\right) & \text { if } m=n=0, \\
A_{m}^{\mathrm{TE}} \frac{L}{2} \operatorname{coth}\left(\gamma_{2 m} d\right) & \text { if } m=n>0,\end{cases}
\end{aligned}
$$

where

$$
\begin{gathered}
L_{m, n}=\int_{-\infty}^{\infty} \frac{1}{\alpha_{1}} \mathcal{F}\left(\cos \left(\frac{n \pi}{L} x\right)\right) \overline{\mp\left(\cos \left(\frac{m \pi}{L} x\right)\right)} \frac{Q_{B}^{\mathrm{TE}}}{\mathcal{Q}_{A}^{\mathrm{TE}}} d \lambda, \\
\mathcal{Q}_{A}^{\mathrm{TE}}=e^{-\alpha_{1} a}\left(\varepsilon_{0} \alpha_{1}-\varepsilon_{1} \alpha_{0}\right)-e^{\alpha_{1} a}\left(\varepsilon_{0} \alpha_{1}+\varepsilon_{1} \alpha_{0}\right), \\
Q_{B}^{\mathrm{TE}}=e^{-\alpha_{1} a}\left(\varepsilon_{0} \alpha_{1}-\varepsilon_{1} \alpha_{0}\right)+e^{\alpha_{1} a}\left(\varepsilon_{0} \alpha_{1}+\varepsilon_{1} \alpha_{0}\right) .
\end{gathered}
$$

The Fourier coefficients, $A_{n}^{\mathrm{TE}}$, form a system of equations that can be solved for computationally by truncating the infinite system to a suitably large finite system.

\section{Use in Finite Element Methods}

The Fourier approach in the previous sections clearly only works when dealing with rectangular cavities. For cavities with arbitrary shape other methods such as the finite element method must be employed [2]. Application of the finite element method finds a solution within a space of functions for the weak form of the Helmholtz equation. Typically the cavity region is approximated by triangular grid and linear tetrahedral functions defined on the grid for a basis for a space of piecewise linear functions. Boundary conditions must be provided in order to solve the problem on a finite region. Therefore, a transparent boundary condition at the cavity opening must be used to properly represent the transmitted field's continuity with the total fields in the regions above it. 
Equations (6.27) and (7.6) can be used to represent such a boundary condition for each of the respective polarizations. Equation (6.27) from the TM case can be viewed as a solution of

$$
\frac{\partial u_{2}}{\partial y}=\frac{\mu_{2}}{\mu_{1}} \mathcal{F}^{-1}\left(\frac{Q_{B}^{\mathrm{TM}}}{\mathcal{Q}_{A}^{\mathrm{TM}}} \mathcal{F}\left(u_{2}\right)\right)+\frac{\mu_{2}}{\mu_{1}} \frac{\partial u^{i r}}{\partial y}
$$

at $y=0$. Such an expression is known as a Dirichlet to Neumann map. It connects the derivative values of $u_{2}$ with the function values. In the Fourier case presented here, the equation is solved by substituting in the Fourier representation of $u_{2}$ and using orthogonality to produce a system of equations. However, (8.1) can be plugged directly into the boundary term of the weak form of the Helmholtz equation. On the other hand, (7.6) from the TE case can be written as

$$
v_{2}=\mathcal{F}\left(\frac{Q_{B}^{\mathrm{TE}}}{\mathcal{Q}_{A}^{\mathrm{TE}}} \mathcal{F}^{-1}\left(\frac{\epsilon_{2}}{\epsilon_{1}} \frac{\partial v_{2}}{\partial y}\right)\right)+v^{i r}
$$

at $y=0$. In this case, the expression is a Neumann to Dirichlet map. Again, in the Fourier case, (8.2) is solved by inserting the Fourier representation of $v_{2}$ and using orthogonality to produce a system of equations. For finite elements in the TE case (8.2) cannot be plugged directly into the weak form of the Helmholtz equation. However, it can be used as auxiliary equation for a mixed finite element method approach.

\section{Far Field Computation}

Computation of far field or radar cross section (RCS) quantities is a postprocessing step. We will use the computed transmitted fields $u_{2}$ and $v_{2}$ above to compute the strength of the scattered fields at distance far from the cavity opening. The trick is that to use the standard formula for far field values, we need to have the scattered field at the interface with free space. That is we need to know the values of $u_{0}^{s}$ and $v_{0}^{s}$ at $y=a$. The far field formulas are [14]

$$
\begin{aligned}
& \sigma_{\mathrm{TM}}\left(\phi, \phi^{i}\right)=\frac{k_{0}}{2}\left|\int_{-\infty}^{\infty} u_{0}^{s}\left(x^{\prime}, a\right) \sin \phi e^{\imath k_{0}\left(x^{\prime} \cos \phi+a \sin \phi\right)} d x^{\prime}\right|^{2}, \\
& \sigma_{\mathrm{TE}}\left(\phi, \phi^{i}\right)=\frac{k_{0}}{2}\left|\int_{-\infty}^{\infty} \frac{i}{\eta \epsilon \omega} \frac{\partial v_{0}^{s}}{\partial y}\left(x^{\prime}, a\right) e^{\imath k_{0}\left(x^{\prime} \cos \phi+a \sin \phi\right)} d x^{\prime}\right|^{2} .
\end{aligned}
$$

The computation of these integrals can potentially be difficult since they are over the entire real line. However, if we relate the scattered fields back to the transmitted fields $u_{2}$ and 
$v_{2}$ in the cavity we can avoid evaluating such an improper integral. If we rewrite above we see that

$$
\begin{aligned}
& \sigma_{\mathrm{TM}}\left(\phi, \phi^{i}\right)=\frac{k_{0}}{2}\left|\sin \phi e^{\imath k_{0}(a \sin \phi)} \int_{-\infty}^{\infty} u_{0}^{s}\left(x^{\prime}, a\right) e^{\imath k_{0}\left(x^{\prime} \cos \phi\right)} d x^{\prime}\right|^{2}, \\
& \sigma_{\mathrm{TE}}\left(\phi, \phi^{i}\right)=\frac{k_{0}}{2}\left|\frac{i}{\eta \epsilon \omega} e^{\imath k_{0}(a \sin \phi)} \int_{-\infty}^{\infty} \frac{\partial v_{0}^{s}}{\partial y}\left(x^{\prime}, a\right) e^{\imath k_{0}\left(x^{\prime} \cos \phi\right)} d x^{\prime}\right|^{2} .
\end{aligned}
$$

We can now see that the integrals are actually Fourier transforms of $u_{0}^{s}$ and $\partial v_{0}^{s} / \partial y$. In Sections 5 and 6 , these Fourier transforms are also expressed as $D_{\mathrm{TM}}(\lambda) e^{-\alpha_{0} a}$ and $-\alpha_{0} D_{\mathrm{TE}}(\lambda) e^{-\alpha_{0} a}$ :

$$
\begin{aligned}
\sigma_{\mathrm{TM}}\left(\phi, \phi^{i}\right) & =\frac{k_{0}}{2}\left|\sin \phi e^{\imath k_{0}(\operatorname{asin} \phi)} D_{\mathrm{TM}}(\lambda) e^{-\alpha_{0} a}\right|^{2}, \\
\sigma_{\mathrm{TE}}\left(\phi, \phi^{i}\right) & =\frac{k_{0}}{2}\left|\frac{i}{\eta \epsilon \omega} e^{\imath k_{0}(\operatorname{asin} \phi)} \alpha_{0} D_{\mathrm{TE}}(\lambda) e^{-\alpha_{0} a}\right|^{2} .
\end{aligned}
$$

Recall from (6.19) that we have from the TM case that

$$
D_{\mathrm{TM}}(\lambda) e^{-\alpha_{0} a}=\frac{2 \mu_{0} \alpha_{1} \sum_{n=1}^{\infty} A_{n}^{\mathrm{TM}} \mathcal{F}(\sin ((n \pi / L) x))}{\mathcal{Q}_{A}^{\mathrm{TM}}}
$$

and a similar calculation in the TE case will give

$$
\alpha_{0} D_{\mathrm{TE}}(\lambda) e^{-\alpha_{0} a}=\frac{2 \epsilon_{0} \epsilon_{1} \sum_{n=1}^{\infty} \gamma_{2} A_{n}^{\mathrm{TE}} \mathcal{F}(\sin ((n \pi / L) x))}{Q_{A}^{\mathrm{TE}}}
$$

These equations can be substituted into (9.3). Hence, the RCS can be evaluated using the computed solutions $u_{2}$ and $v_{2}$ from inside the cavity.

\section{Fast RCS Approximation}

Morgan and Schwering presented a simple method for rapidly producing approximations of the RCS for filled rectangular cavities. A mode matching technique replaces the open half space above the cavity with a vertical channel containing outgoing waveguide modes. The coefficients are computed directly without building and solving a linear system by matching the cavity modes with the outgoing waveguide modes [4]. The approach yields surprisingly good agreement with the true solutions when used for RCS computations. Bao and Zhang attempted to refine the mode matching approach by improving the approximation using an asymptotic argument [3]. The "impoved" mode matching solution converges to the exact solution as the frequency increases. 


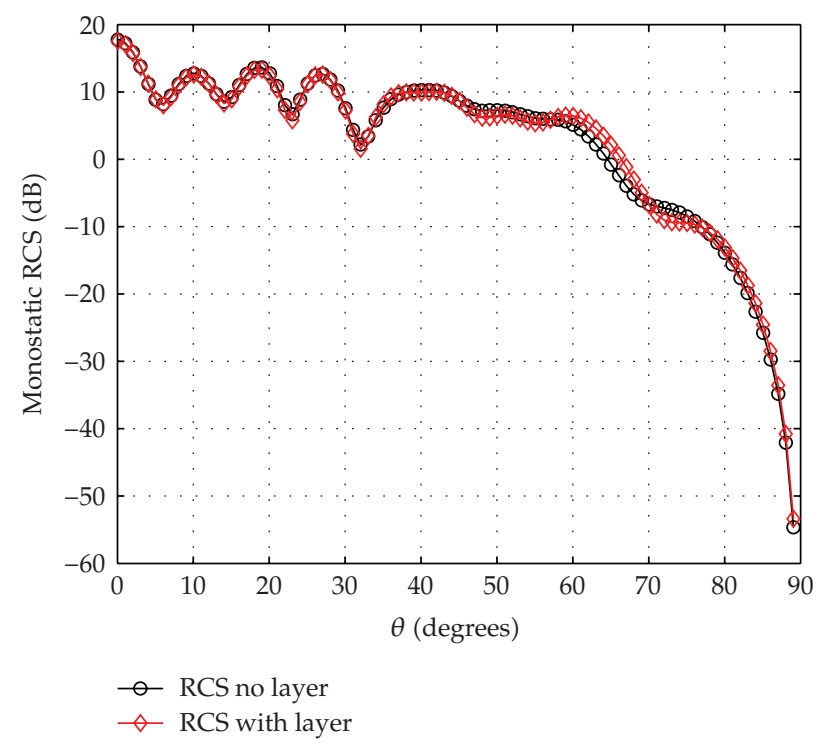

Figure 2: Thin layer perturbation for the TM case.

The fast mode matching approximation of Morgan and Schwering extends the cavity walls into the upper half space and uses a mode matching solution as an approximation [4]. If the same mode matching approach is applied to an overlayer problem, it would be performed at the interface from the cavity material to the overlayer material and then again from the overlayer material to free space parameters. It is the author's observation based on comparison with [4] that this is essentially equivalent to using only the diagonal terms of (6.27) and (7.6) in the full Fourier derivation and solving the corresponding diagonal system. Although this equivalence is not formally investigated here, it can be seen below that the solution of the diagonal system yields solid results. It is possible that this equivalence is the path to a future formal Mathematical analysis of the convergence properties of the mode matching approach.

\section{Numerical Results}

The following results demonstrate that the derivations are sound and show the efficacy of the method. As was done in [4], the parameters are chosen arbitrarily for demonstration purposes.

\subsection{Basic Computations}

Figures 2, 3, 4, 5, 6, 7, 8 and 9 show the solutions obtained by solving (6.27) for the TM case and 19 for the TE case. Since there are no results to compare with directly, the output is validated by physical interpretation. First, a cavity underneath a very thin layer of dielectric material is compared to the same cavity with no dielectric overlayer. Note that the layered problem results in a small perturbation of the solution of the nonlayered problem as one would expect. The cavity geometry is given by $L=1$ and $d=10$. The material parameters are 


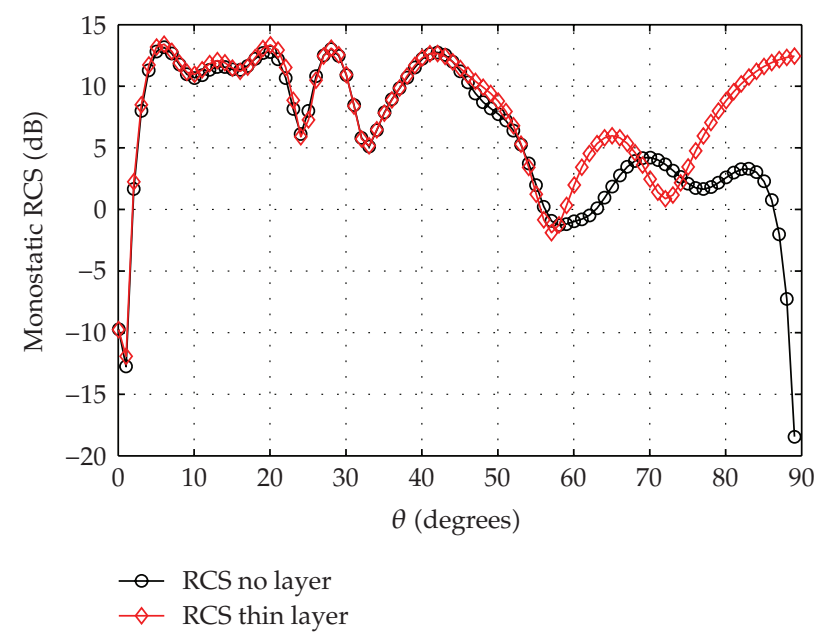

Figure 3: Thin layer perturbation for the TE case.

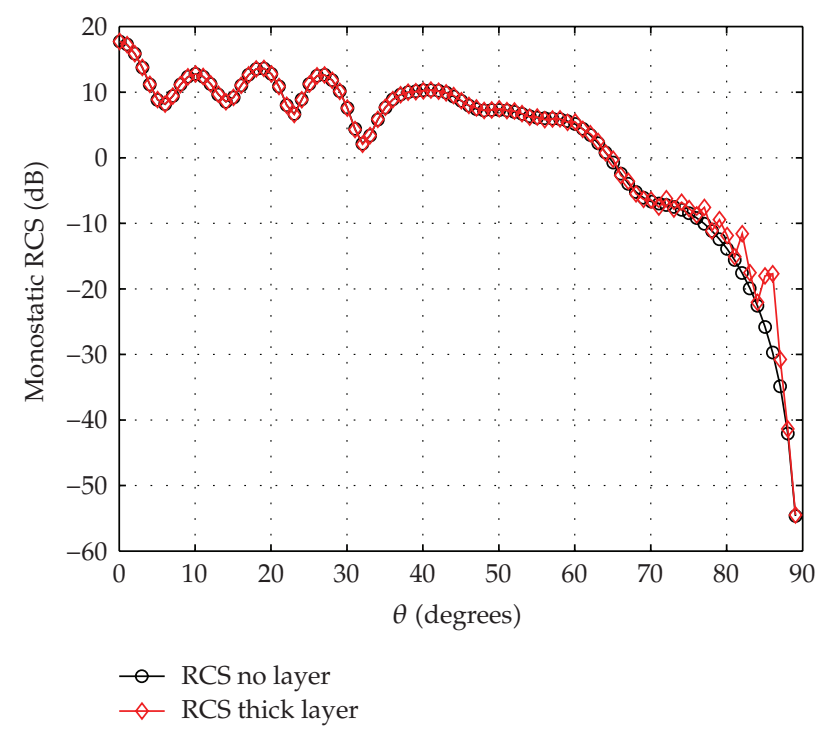

Figure 4: Thick layer perturbation for the TM case.

given by $\epsilon_{1}=2, \mu_{1}=3, \epsilon_{2}=2$ and $\mu_{2}=1$ and the frequency is $14 \pi$. The layer has thicknes $a=.1$. The computation of a monostatic radar cross section is given for both the TE and TM cases.

Note that for angles near 90 degrees we may expect more prominent disagreement. The deviation is due to the fact that the fields will travel through much more material at angles near grazing incidence. The deviation is much more prominent in the TE example for Figure 3 due to the presence of surface waves traveling in the overlayer dielectric medium. In the TM case, far fields tend to zero near grazing incidence which explains the better agreement for Figure 2 [15]. 


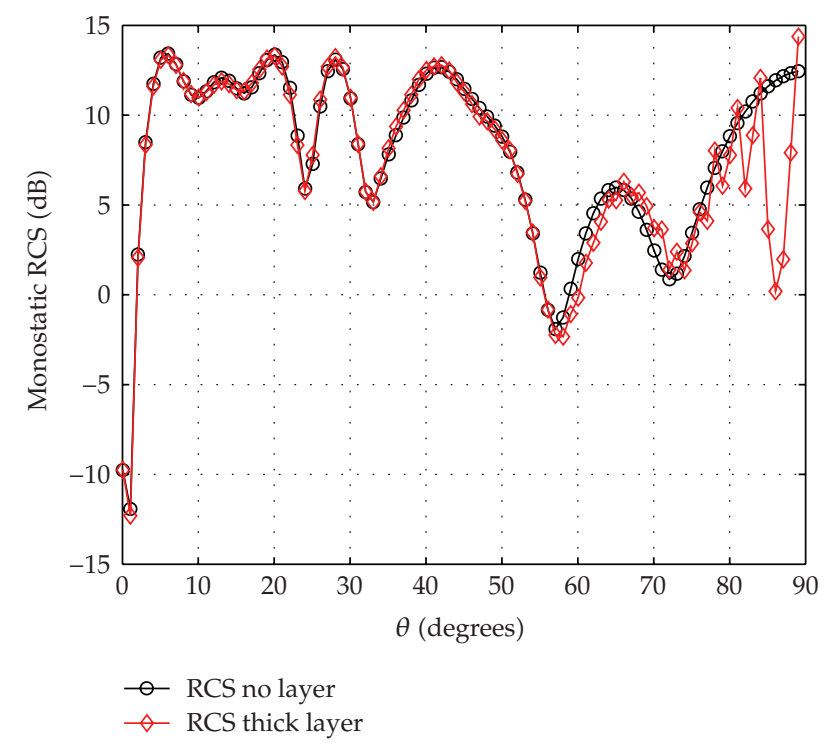

Figure 5: Thick layer perturbation for the TE case.

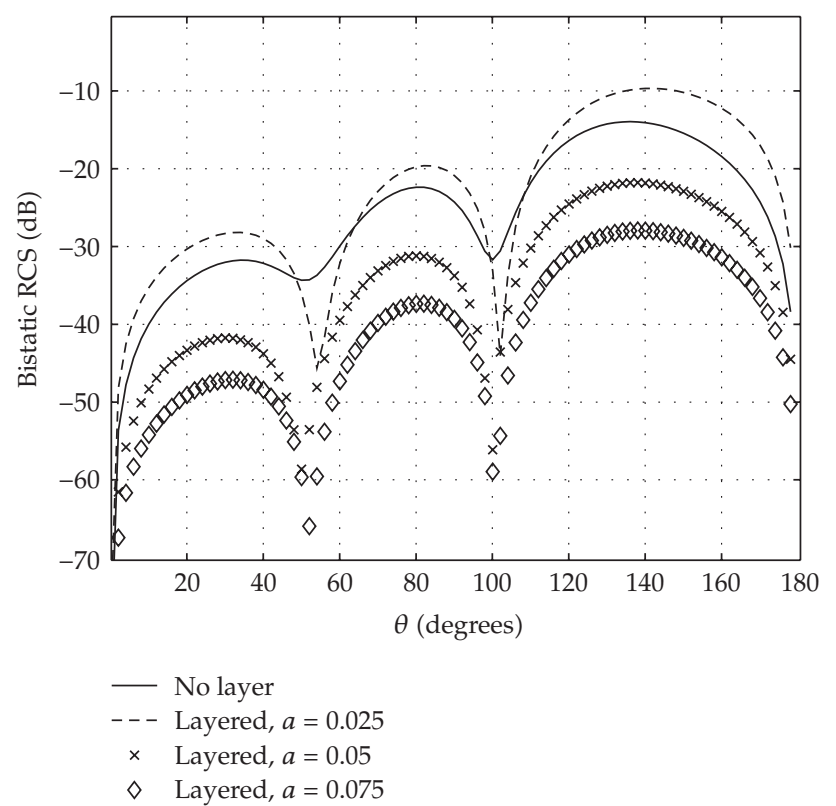

Figure 6: Increasing layer of conducting material.

A second example deals with an thicker overlayer but with a only a small change in the dielectric materials. Again, as one would expect only a small perturbation of the solution is seen. A similar explanation as given above regarding Figure 3 explains the deviations found near 90 degrees. The cavity geometry is the same as above but the material parameters are given by $\epsilon_{1}=1.01, \mu_{1}=1.01, \epsilon_{2}=2$, and $\mu_{2}=1$. The thickness of the overlayer material is $a=1$. The frequency is also the same as above. Note that the material parameters in the layer 


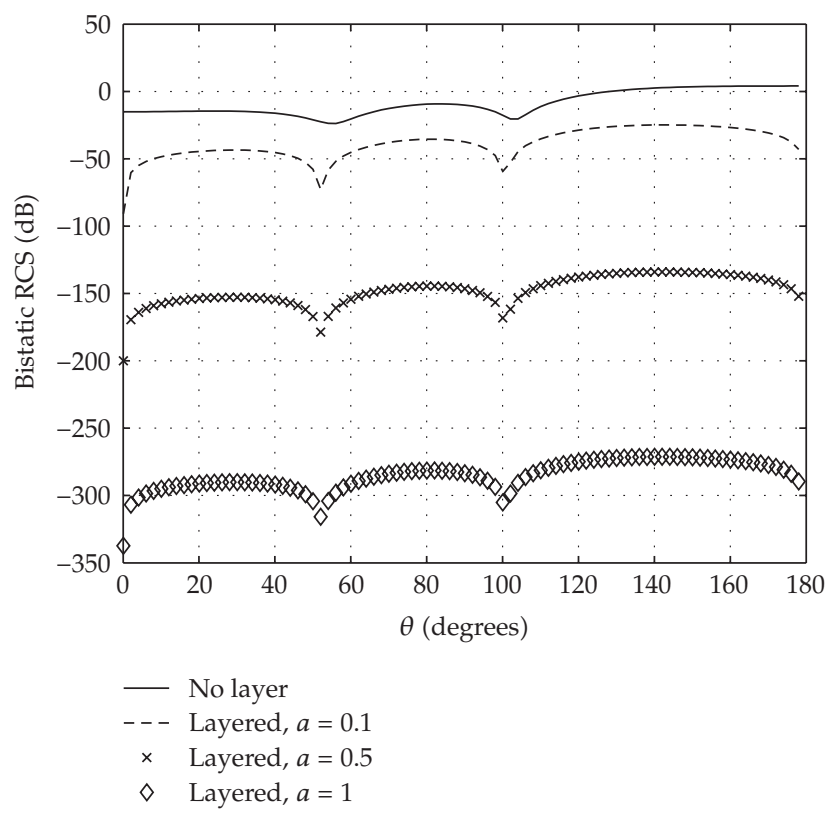

Figure 7: Increasing layer of conducting material.

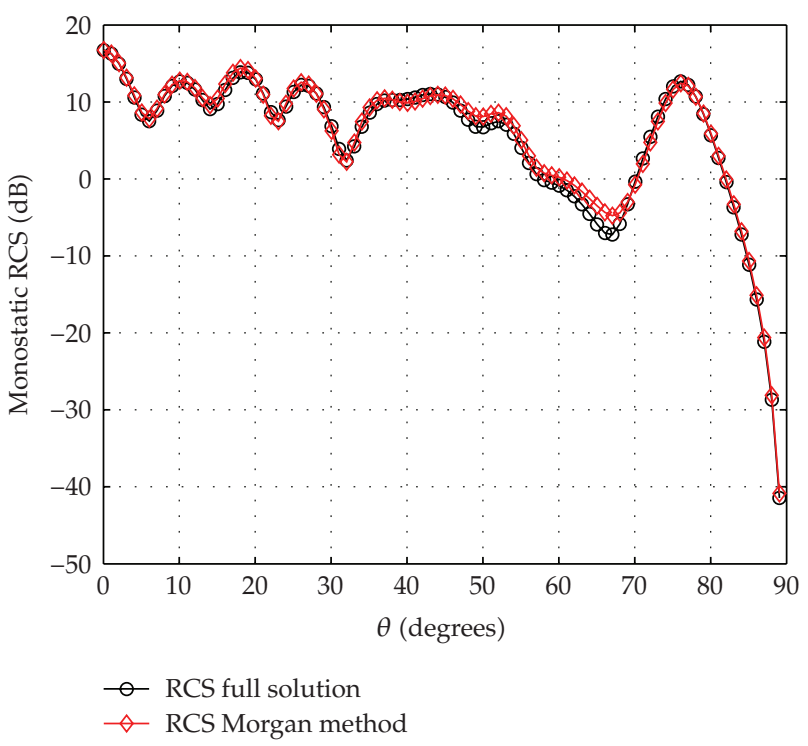

Figure 8: Comparison of full solution with Morgan and Schwering mode matching method for the TM case.

only slightly differ from that of free space. Again the monostatic radar cross section is computed for both the TE and TM cases.

Finally, a computation of bistatic radar cross sections is performed for which the thickness of the layer is increased. The layer material is a conductor which means that as the thickness of the layer increases there is a corresponding decrease in the strength of 


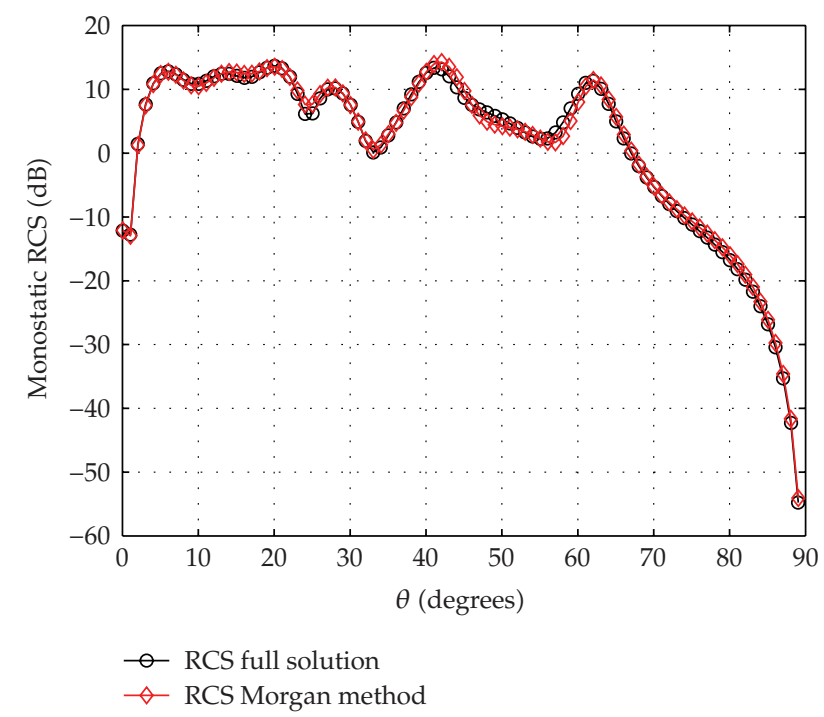

Figure 9: Comparison of full solution with Morgan and Schwering mode matching method for the TE case.

the far field. In this example the geometry is given by $L=1.25$ and $d=.0625$. The material parameters are $\epsilon_{1}=16-5 \imath, \mu_{1}=4-1.25 \imath, \epsilon_{2}=1$ and $\mu_{2}=1$. The frequency is $2 \pi$. Again the results agree with the expected physical interpretation.

\subsection{Fast Approximations}

Here the mode matching approximation of Morgan and Schwering as applied to the layered problem is demonstrated. The comparisons between the mode matching approximation and the full solution agree well. Although it has not been explained mathematically, the numerical experiments show that the mode matching method appears to work effectively with electrically large deep cavities. Some mathematical explanation is given by Bao and Zhang [3]. The geometry used here is again $L=1$ and $d=10$. The material parameters are $\epsilon_{1}=2, \mu_{1}=3, \epsilon_{2}=2$ and $\mu_{2}=1$. The frequency is $14 \pi$.

The agreement between the full and mode matching solutions is remarkable given the time savings. The full problem requires a full $N \times N$ matrix to be built and the corresponding system to be solved. The mode matching approximation used only the diagonal terms of the system and hence needs only to compute $N$ matrix entries and solve a diagonal system. The time saved is obviously quite dramatic. For example, the computation of the results in Figures 8 and 9 took nearly 3000 seconds with the full solution while the mode matching approximation took less than 40 seconds.

\section{Conclusion}

A complete Fourier solution has been provided for the problem of electromagnetic scattering by a recatangular buried cavity beneath a uniform material layer. It has been demonstrated that the method gives solutions that agree with physical interpretations. Also, the mode matching method described by Morgan and Schwering has been extended to the buried 
cavity problem. The mode matching technique works very well in this context and provides enormous speed up in terms of computation.

The technique can be extended to rectangular cavities buried under multiple uniform material layers. It will also be applied in finite element techniques as a transparent boundary condition for similar problems involving nonrectangular cavities. In the future, it would be very useful to extend the approach to three dimensional cavities. Also, a thorough mathematical investigation of the convergence properties of the mode matching technique would be desirable.

\section{References}

[1] G. W. Hanson and A. B. Yakovlev, Operator Theory for Electromagnetics: An Introduction, Springer, New York, NY, USA, 2002.

[2] T. Van and A. W. Wood, "Finite element analysis of electromagnetic scattering from a cavity," IEEE Transactions on Antennas and Propagation, vol. 51, no. 1, pp. 130-137, 2003.

[3] G. Bao and W. Zhang, "An improved mode-matching method for large cavities," IEEE Antennas and Wireless Propagation Letters, vol. 4, no. 1, pp. 393-396, 2005.

[4] M. A. Morgan and F. K. Schwering, "Mode expansion solution for scattering by a material filled rectangular groove," Progress in Electromagnetics Research, vol. 98, no. 18, pp. 1-17, 1998.

[5] J. Jin, The Finite Element Method in Electromagnetics, Wiley-IEEE Press, New York, NY, USA, 2nd edition, 2002.

[6] T. J. Park, H. J. Eom, and K. Yoshitomi, “An analytic solution for transverse-magnetic scattering from a rectangular channel in a conducting plane," Journal of Applied Physics, vol. 73, no. 7, pp. 3571-3573, 1993.

[7] T. J. Park, H. J. Eom, and K. Yoshitomi, "An analysis of TE-scattering from a rectangular channel in a conducing plane," Radio Science, vol. 28, no. 5, pp. 663-673, 1993.

[8] K. Barkeshli and J. L. Volakis, "Scattering by an aperature formed by a rectangular cavity in a ground plane," Tech. Rep. 389757-2-T, University of Michigan Radiation Laboratory, Ann Arbor, Mich, USA, 1989.

[9] D. J. Hoppe and Y. Rahmat-Samii, Impedance Boundary Conditions in Electromagnetics, Taylor \& Francis, Washington, DC, USA, 1995.

[10] E. Howe, Analysis and numerical solution of an integral equation method for electromagnetic scattering from a cavity in a ground plane, M.S. thesis, Air Force Institute of Technology, Ohio, Ohio, USA, April 2001.

[11] W. Wood, Electromagnetic scattering from a cavity in a ground plane: theory and experiment, Ph.D. thesis, Air Force Institute of Technology, Ohio, Ohio, USA, 1999.

[12] A. Wood, "Analysis of electromagnetic scattering from an overfilled cavity in the ground plane," Journal of Computational Physics, vol. 215, no. 2, pp. 630-641, 2006.

[13] J. L. Fleming, "Convergence analysis of a Fourier-based solution method of the Laplace equation for a model of magnetic recording," Mathematical Problems in Engineering, vol. 2008, Article ID 154352, 11 pages, 2008.

[14] A. F. Peterson, S. L. Ray, and R. Mittra, Computational Methods for Electromagnetics, IEEE/OUP Series on Electromagnetic Wave Theory, IEEE Press, New York, NY, USA, 1998.

[15] R. E. Collin, Field Theory of Guided Waves, Wiley-IEEE Press, New York, NY, USA, 1990. 


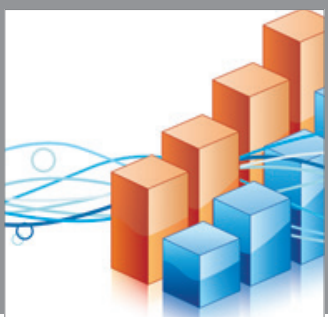

Advances in

Operations Research

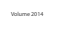

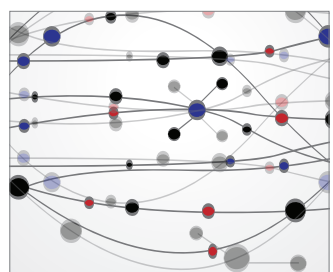

\section{The Scientific} World Journal
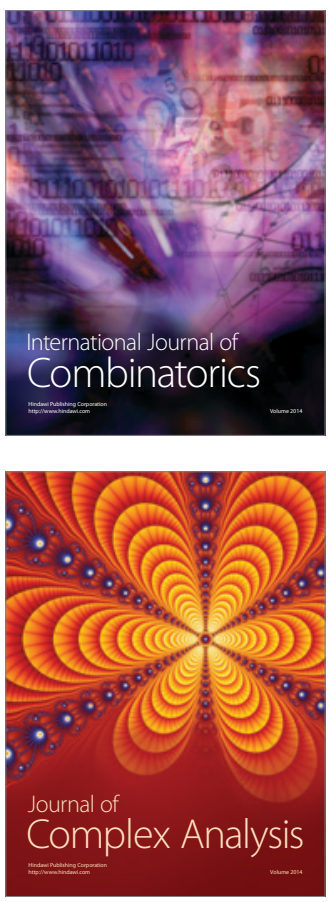

International Journal of

Mathematics and

Mathematical

Sciences
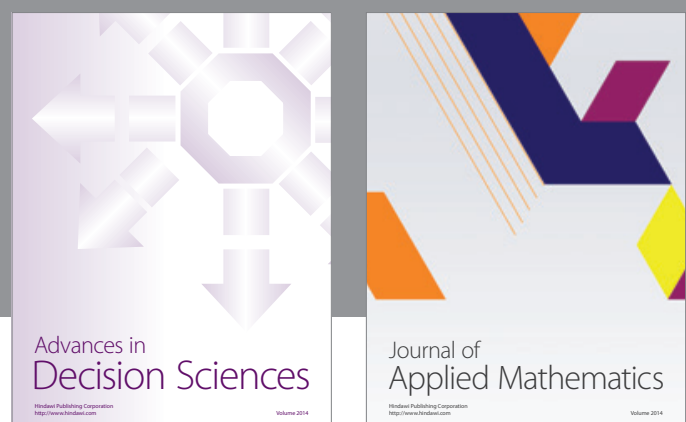

Journal of

Applied Mathematics
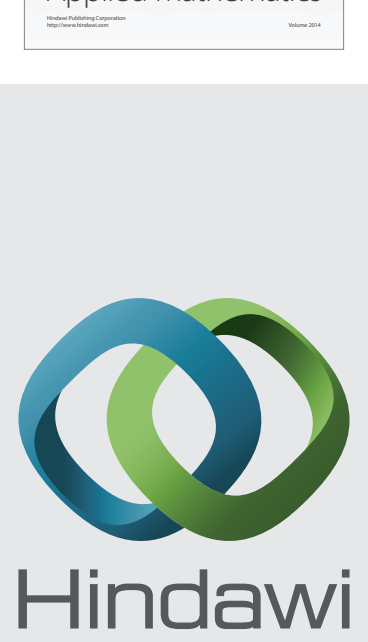

Submit your manuscripts at http://www.hindawi.com
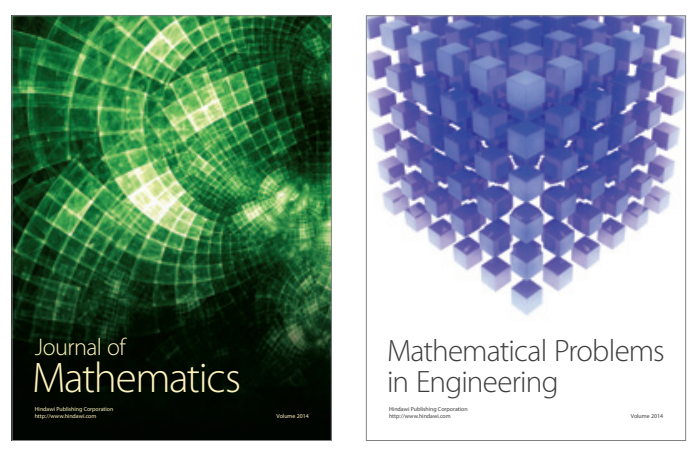

Mathematical Problems in Engineering
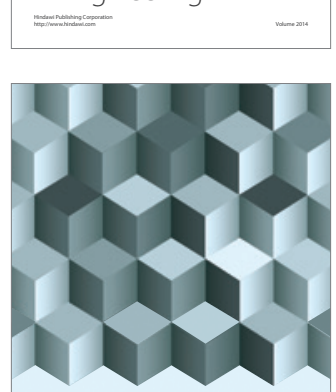

Journal of

Function Spaces
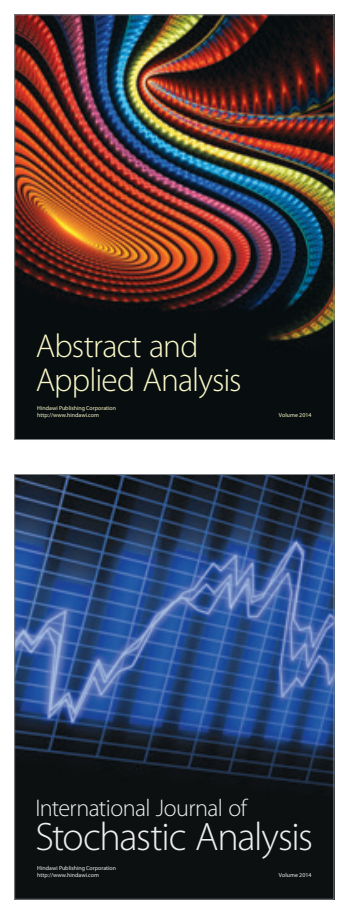

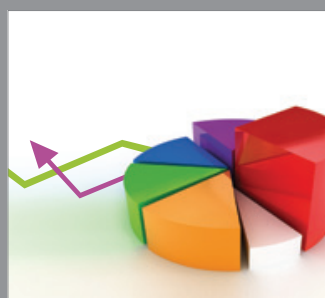

ournal of

Probability and Statistics

Promensencen
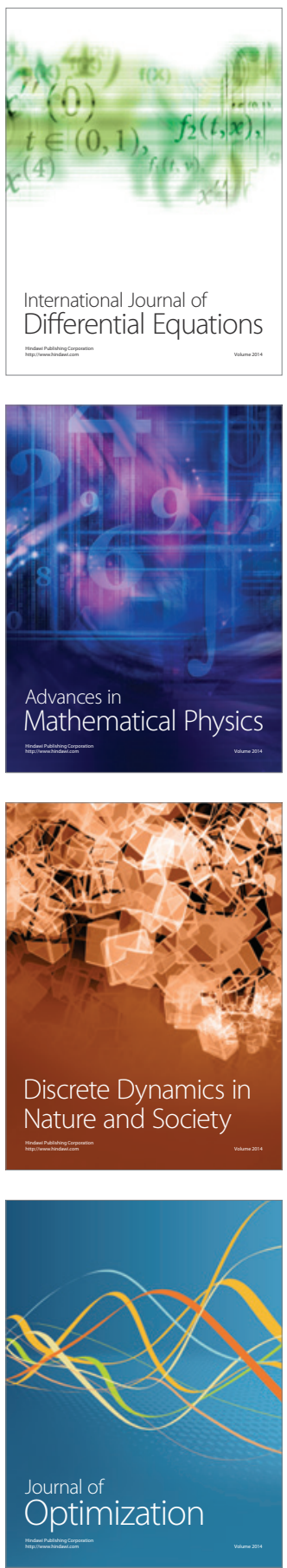